La revue La revue pour l'histoire du CNRS

$2 \mid 2000$

Les premiers laboratoires du CNRS

\title{
Pierre Curie, 1859-1906. Le rêve scientifique
}

Loïc Barbo. Préface d'Hélène Langevin-Joliot, Belin, Collection « Un savant, une époque ", Paris, 1999

Michel Paty

\section{(2) OpenEdition}

\section{Journals}

Édition électronique

URL : https://journals.openedition.org/histoire-cnrs/374

DOI : $10.4000 /$ histoire-cnrs. 374

ISSN : 1955-2408

Éditeur

CNRS Éditions

\section{Édition imprimée}

Date de publication : 5 mai 2000

ISBN : 978-2-271-05708-2

ISSN : 1298-9800

\section{Référence électronique}

Michel Paty, «Pierre Curie, 1859-1906. Le rêve scientifique », La revue pour l'histoire du CNRS [En ligne], 2 | 2000, mis en ligne le 06 mars 2006, consulté le 20 mai 2021. URL : http://journals.openedition.org/ histoire-cnrs/374 ; DOI : https://doi.org/10.4000/histoire-cnrs.374

Ce document a été généré automatiquement le 20 mai 2021.

Comité pour l'histoire du CNRS 


\title{
Pierre Curie, 1859-1906. Le rêve scientifique
}

\author{
Loïc Barbo. Préface d'Hélène Langevin-Joliot, Belin, Collection « Un \\ savant, une époque ", Paris, 1999
}

\section{Michel Paty}

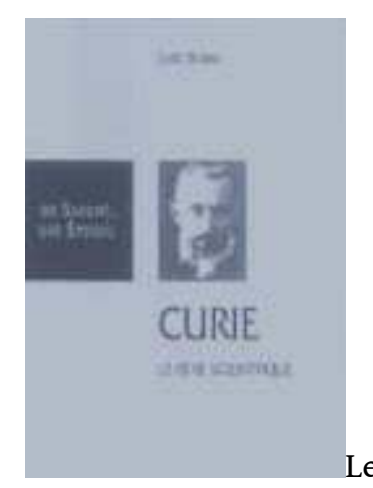

Le livre que Loïc Barbo vient de publier sur Pierre Curie, fruit d'une thèse soutenue en 1998 à l'université de Nantes, est assurément appelé à faire date. Par sa nécessité, tout d'abord, les biographies de ce savant discret et pourtant très connu étant fort rares. Sa vie, inséparable de celle de son épouse Marie, née Sklodowska, était surtout évoquée dans les ouvrages consacrés à cette dernière (rappelons la biographie par Robert Reid, publiée en traduction française voici plus de vingt ans ${ }^{1}$ ). Marie Curie avait rédigé à la mémoire de son mari une émouvante évocation, parue en $1923^{2}$, et l'on ne comptait jusqu'ici qu'une seule biographie due à la plume d'un non-familier, celle, récente, écrite par Anna Hurwic ${ }^{3}$.

1 L'ouvrage de Loïc Barbo s'impose, ensuite, par sa richesse d'informations, sa clarté, ses qualités de compréhension scientifique et historique, et de finesse d'analyse. Aucun aspect important de la vie, de l'œuvre et de la pensée de Pierre Curie n'est laissé dans l'ombre, et l'auteur nous fait rencontrer le chercheur, le penseur scientifique, mais également l'homme, restitué dans le contexte historique qui fut le sien, la France de la IIIe République, avec ses institutions d'enseignement et de recherche, dont les traits essentiels sont rappelés (universités et grandes Écoles, contenus des programmes et conception des thèses en physique, etc.), au sein desquelles le savant eut à lutter au 
long d'une carrière qui ne fut jamais facile, jusqu'à sa mort prématurée, en 1906, à l'âge de quarante-sept ans.

2 La méthode d'exposition choisie combine de façon souple l'ordre thématique, qui favorise la compréhension et l'unité narrative, tantôt portant l'accent sur les contenus scientifiques et tantôt sur les milieux, et l'ordre chronologique des événements. Le souci d'exactitude dans la documentation et la rigueur dans l'exposé des faits mis en situation, qui garantissent la qualité de ce travail d'historien, ne nuisent en rien à l'intérêt que le lecteur prend à ce récit d'une vie, qui se lit, à vrai dire, comme un roman, au contenu fort substantiel.

Le profil d'une personnalité - son caractère, ses idées, qui, au fil des événements, tant individuels que collectifs et sociaux, vont se cristalliser en une œuvre et en une destinée - est préparé (mais non pas, bien entendu, déterminé !) par l'enfance et par les années de formation. La famille, dans le cas de Pierre Curie, joua un grand rôle, et l'on retient la tradition du côté paternel (sans remonter à l'origine lointaine des Curie, ce qui ferait traverser la France et son histoire depuis les Guerres de religion qui, du sud, les chassèrent au nord-est, à Montbéliard), avec les figures marquantes du grand-père et du père, médecins, acquis aux idées sociales (Paul, le grand-père, était saintsimonien, Eugène, le père, s'était fait mal voir de sa clientèle bourgeoise par son engagement dans la Commune de Paris), adeptes de la médecine homéopathique, et de convictions républicaines et laïques (Pierre et son frère aîné Jacques ne reçurent aucune éducation religieuse).

Eugène Curie éleva ses deux fils dans cet idéal, et leur transmit son goût de la nature, son culte de la science (dans un scientisme bien de l'époque) et la vocation de la recherche (la sienne avait été contrariée par la nécessité de faire vivre sa famille en exerçant la profession de médecin scolaire ou de quartier, humble et dévoué à ses patients). Veuf en 1897, il demeura quelque temps dans la famille de Pierre avant de retourner vivre à Sceaux, où il mourut en 1910, quelques années après avoir eu l'immense douleur de pleurer la mort accidentelle du fils bien-aimé. La présence discrète de ce père se laisse sentir tout au long des événements de la vie de Pierre Curie, et cette marque du contexte familial se retrouve aussi dans les relations toujours étroites entre les deux frères, Jacques et Pierre, qui travaillèrent ensemble, à leurs débuts, sur les phénomènes électriques liés aux cristaux, découvrirent (en 1880) et étudièrent la piézo-électricité (déformation mécanique des cristaux sous l'influence de forces électriques), et mirent au point des appareils de mesure de précision utilisant ce nouveau phénomène.

5 La formation intellectuelle de Pierre Curie fut aussi fortement marquée par ses rapports avec le "réseau des chimistes alsaciens ", qui avaient rejoint Paris pour fuir leur région annexée en 1871 par l'expansion prussienne, et qui avaient pour nom Charles Lauth, Paul Schutsenberger, Charles Friedel, Albin Haller, eux-mêmes disciples d'Adolphe Wurtz, partisan résolu des conceptions atomistes (lesquelles comptaient alors surtout des adversaires influents). Pierre Curie partagea avec eux ces convictions, qui lui furent essentielles dans son travail scientifique. L'année même où fut créée, sous l'influence de ces chimistes, l'École municipale de physique et de chimie industrielle de la ville de Paris (une école alors sans équivalent en France, et qui devait être, comme on le sait, d'un grand prestige), il y fut admis comme "préparateur », en 1882, gravissant ensuite les échelons de la carrière pendant les vingt-deux années où il y enseigna, ne devenant professeur qu'en 1898 avant de connaitre, très tardivement, peu avant sa 
mort accidentelle (en 1906), la consécration d'une chaire à la Sorbonne, en 1904 et, enfin, celle de l'Académie des sciences, en 1905.

6 La tâche principale qu'exerça Pierre Curie fut celle d'enseignement, mais il était porté par la «passion de la recherche », que les structures institutionnelles de l'époque ne lui facilitèrent en rien. Il fut reconnu par les savants étrangers les plus prestigieux avant de l'être dans son propre pays (la faute aux hiérarchies d'écoles). Lord Kelvin, en visite chez le jeune chercheur français qui venait de découvrir la piézo-électricité, s'étonnait de la soupente précaire qui lui servait de laboratoire...

N'eût été l'éclat public que lui valurent ses découvertes sur la radioactivité affectées avec Marie, sa femme, avec qui il voulut partager son « rêve scientifique », Pierre Curie, chercheur génial et modeste, aurait pu être l'un de ces savants que la postérité a plus ou moins oublié, malgré des apports scientifiques considérables. Ceux de la première période sa vie de chercheur furent d'une importance majeure pour la physique tant expérimentale que théorique: outre la piézo-électricité, il étudia, dès 1884, les symétries des phénomènes physiques (son expérience de cristallographe contribua à l'y orienter), inaugurant pour la physique des considérations qui devaient se révéler de très grande portée. Ces travaux le montrent comme un physicien théoricien dans la lignée d'un Ludwig Boltzmann, d'un Marcel Brillouin, comme le serait aussi un Paul Langevin, pour qui la théorie physique part des lois des phénomènes eux-mêmes pour en donner une représentation de nature fondamentale, gouvernée par des principes généraux (de symétrie dans son cas). Le troisième volet de cette partie de son œuvre est l'étude des phénomènes magnétiques (menée de 1891 à 1895), en particulier leur comportement avec la température, recherches qui constituent le sujet de sa thèse, et qui devaient servir de base à la théorie atomique des différentes formes du magnétisme (dia-, para-, ferro-magnétismes), dont un premier état fut donné dès 1905 par Paul Langevin puis par Pierre Weiss. L'année de sa thèse (1895) fut aussi celle de son mariage avec Marie Sklodowska, avec laquelle il se voua, à partir de 1898, à l'étude de la radioactivité découverte deux années auparavant par Henri Becquerel. Cette aventure, qui leur valut (aux trois) le prix Nobel de l'année 1903, est mieux connue, mais ce n'est pas le moindre mérite du livre de Loïc Barbo (que cette date partage en deux) de nous raconter de manière très précise leur participation respective. Un nouveau continent des phénomènes de la nature fut ainsi révélé, dont ils furent les premiers explorateurs, avec la découverte et l'identification de radioéléments jusqu'alors inconnus, le polonium et le radium, et la mise en évidence de nombreuses propriétés de ces substances, y compris leurs effets biologiques... Ce fut une période de recherches fiévreuses, sans grands moyens (la big science ne viendrait qu'après), suivie d'une reconnaissance mondiale qui ne modifia en rien les habitudes et la modestie du savant. Puis l'accident brutal, imprévisible, absurde, venu sans explication mettre un terme à cette vie, à une histoire humaine, l'une de celles dont l'histoire est faite... 


\section{NOTES}

1. Robert Reid, Marie Curie, Seuil, Paris 1979.

2. Marie Curie, Pierre Curie, Payot, Paris, 1923 ; Denoël, Paris 1955 ; Odile Jacob, Paris 1996.

3. Anna Hurwic, Pierre Curie, Flammarion, Paris, 1995.

\section{AUTEUR}

\section{MICHEL PATY}

Directeur de recherche au CNRS, équipe REHSEIS, CNRS - université de Paris VII 